\title{
Pulmonary sequestrations: Experience of 31 patients
}

\author{
Ersin Sapmaz ${ }^{1}$, Merve Şengül İnan ${ }^{1}$, Hakan Işık ${ }^{1}$, Okan Karataş ${ }^{2}$, Hasan Çaylak ${ }^{1}$, Sedat Gürkök ${ }^{1}$
}

(1) Gulhane Training and Research Hospital, Department of Thoracic Surgery, Ankara, Turkey

(2) Sinop Atatürk State Hospital, Department of Thoracic Surgery, Sinop, Turkey

Date submitted:

May 08, 2019

Date accepted:

May 16, 2019

Online publication date:

June 15, 2019

\section{Corresponding Author: \\ Ersin Sapmaz \\ Gulhane Training and Research \\ Hospital, Department of \\ Thoracic Surgery, Ankara, \\ Turkey \\ dr.esapmaz@gmail.com}

Keywords: Pulmonary sequestration, extralobar sequestration, intralobar sequestration, pulmonary congenital malformation, thoracic surgery.

\begin{abstract}
Aims:Pulmonary sequestration (PS) is a rare congenital lung malformation. It is characterized by a nonfunctional pulmonary tissue that have no relation to the bronchial system. Case series are rare due to low incidence of pulmonary sequestration. In this study, we aim to analyze the presentation, demographics, diagnostic procedures, location, type and treatment of PS in our institution over 12-year period.

Methods:We retrospectively evaluated medical records of pathologically proved PS from January 2003 through December 2015 in our institution. Thirty-one patients were included in the study. Demographics, clinical presentation, diagnostic imaging, location, type of sequestration, type of surgery were collected.

Results:Of 31 patients, 3 patients (9.6\%) were female and 28 patients were (90.3\%) male. Average age was 24.6 years. Twenty-four (77\%) patients had described symptoms. Chest X-ray scanning was performed to all patients as the first imaging modality. Of the 15 patients $(48.4 \%)$ had intralobar sequestration and 16 patients (51.6\%) had extralobar sequestration. Extralobar sequestrations were more common (55.1\%). Lower lobectomy in 13 patients, segmentectomy in 2 intralobar sequestration patients, and simple mass excision in all extralobar sequestration patients (16 patients) were performed as surgical procedure. Six patients had postoperative complications: prolonged air leak in 4 patients, pneumonia in 2 patients.

Conclusions:It has similar appearance with many diseases of lung with traditional imaging methods and cause difficulties in diagnosis. Proving the presence of a feeding systemic artery with angiographic imaging is essential for definitive diagnosis. The recommended optimal treatment for pulmonary sequestration is resection of the sequestered tissue by segment or lobar resection.
\end{abstract}

\section{Introduction}

Pulmonary sequestration (PS) is a rare congenital lung malformation with a rate of $0.15-6.4 \%$ (1). It is characterized by a nonfunctional pulmonary tissue that have no relation to the bronchial system. It provides arterial blood supply directly from thoracic or abdominal aorta or from one or several intercostal branches of the aorta (2). Pathogenesis of this rare entity has not clearly understood so far and has not been associated with a chromosomal abnormality yet. There is no consensus on one single embryonic hypothesis. The most common idea is that these lesions appear as accessory bud and move together with the developing esophagus and provides its blood supply from systemic circulation $(3,4)$. This disease is considered in the disease group with normal or abnormal pulmonary tissue with normal or abnormal vascularization.

Two type of PS has been defined: in the lobar parenchyma as intralobar pulmonary sequestration (ILS) and outside of the lob as extralobar pulmonary sequestration (ELS). ILS is more common than ELS (86-75\%) (5). Extralobar sequestrations are distinguished by having their own pleura.
Pulmonary sequestration in adult patients is usually diagnosed after a patient's complaint of symptoms or incidentally when having a health check or thoracic scanning for other reasons.

Case series are rare due to low incidence of pulmonary sequestration. In this study, we aim to analyze the presentation, demographics, diagnostic procedures, location, type and treatment of PS in our institution over 12-year period.

\section{Methods}

We retrospectively evaluated medical records of pathologically proved PS from January 2003 through December 2015 in our institution. We reevaluated all patients with surgery notes and imaging studies. We excluded patients who had only radiologic diagnosis and refused surgical treatment. Thirty-one patients were included in the study. Demographics, clinical presentation, diagnostic imaging, location, type of sequestration, type of surgery were collected. Approval for the study was granted by the Ethics Committee of Gulhane Training and Research Hospital (Approval Date: March 26, 2019, Approval Number: 19/106). 


\section{Results}

Of 31 patients, 3 patients $(9.6 \%)$ were female and 28 patients were $(90.3 \%)$ male. Average age of the patients was 24.6 years, ranging between 12 and 50 years. There were no children, two of patients were at adolescent age, 12 and 16 years. Twenty-nine adults were between $20-50$ years (Table 1 ).

\begin{tabular}{lcc|}
\hline $\begin{array}{l}\text { Table 1. Demographic distribution of pulmonary } \\
\text { sequestration patients }(\mathbf{n = 3 1 )}\end{array}$ & $\mathbf{n}$ \\
\hline & & \\
\hline Gender & 28 & 90.3 \\
\hline Male & 3 & 9.7 \\
\hline Female & & \\
\hline Age & 0 & 0 \\
\hline Children & 2 & 6.5 \\
\hline Adolescent & 29 & 93.5 \\
\hline Adults & & \\
\hline Presentation & 24 & 77 \\
\hline Symptomatic & 10 & 42 \\
\hline - Dyspnea & 7 & 29 \\
\hline - Recurrent episodes of pneumonia & 5 & 21 \\
\hline - Chest pain & 2 & 8 \\
\hline - Hemoptysis & 7 & 23 \\
\hline Asymptomatic & & \\
\hline
\end{tabular}

Twenty-four (77\%) patients had described symptoms: shortness of breath in 10 patients, recurrent episodes of pneumonia in 7 patients, chest pain in 5 patients, hemoptysis in 2 patients (Table 1).

Chest X-ray scanning was performed to all patients as the first imaging modality, which often showed nonspecific opacities such as mass like density. In 6 patients (19.3\%), Computed Tomography Angiography (CTA) identified the suspicious lesions for pulmonary sequestration by demonstrating a systemic arterial supply to the sequestrated segment. In one patient $(3.2 \%)$, arterial blood supply was diagnosed with an Magnetic Resonance Angiography and in one patient (3.2\%), arterial blood supply was diagnosed with conventional angiography. CTA revealed aberrant arteries in two patients which were presented with hemoptysis. Bronchoscopy was performed to determine the suspected area of hemoptysis in symptomatic patients.

Of the 15 patients (48.4\%) had ILS and 16 patients $(51.6 \%)$ had ELS. There were 15 male (93.7\%) patients and one female (6.3\%) patients in ELS group, and there were 2 female (13.3\%) patients and 13 male $(86.7 \%)$ patients in ILS group (Table 2).

\begin{tabular}{lcc}
\hline \multicolumn{3}{l}{ Table 2. Gender related types of PS } \\
\hline & Female & Male \\
\hline ELS & 1 & 15 \\
\hline ILS & 2 & 13 \\
\hline Total & 3 & 28 \\
\hline
\end{tabular}

Although the rates were similar in adult group (29 patients), extralobar sequestrations were more common (\%55.1). Two adolescent patients' sequestrations were both intralobar type, and located in left lower lobe. In all female patients, sequestrations were located in left lower lobe and two of them were ELS but one was ILS. The most common type in women was ILS, but ELS in men.

In 21 patients $(67.8 \%)$, the PS were located in the left lower lobe and in 8 patients (25.8\%) in the right lower lobe. One PS $(3.2 \%)$ was located in the right middle lobe and one PS $(3.2 \%)$ was located in the left upper lobe.

Half of the sequestrations in the left lower lobe (22 patients, $70.9 \%$ ) were ILS and the other half were ELS. One patient had ILS-type sequestration in the right middle lobe. Of the 8 patients $(25.8 \%)$ in which the sequestration was located in the right lower lobe, 3 of them were (37.5\%) ILS, and 5 of them $(63.5 \%)$ were ELS (Table 3).

\begin{tabular}{llccc}
\hline \multicolumn{3}{l}{ Table 3. Type related localization of PS } \\
\hline \multirow{3}{*}{ Right } & ELS & ILS & Total \\
\hline & Upper lobe & 0 & 0 & \\
\cline { 2 - 5 } & Middle lobe & 0 & 1 & 1 \\
\cline { 2 - 5 } & Lower lobe & 5 & 3 & 8 \\
\hline \multirow{2}{*}{ Left } & Upper lobe & 0 & 0 & \\
\cline { 2 - 5 } & Lower lobe & 11 & 11 & 22 \\
\hline \multirow{2}{*}{ Total } & & 16 & 15 & 31 \\
\hline
\end{tabular}

Lower lobectomy in 13 ILS patients, segmentectomy in 2 ILS patients, and simple mass excision in all ELS patients (16 patients) were performed as surgical procedure. Twentynine of the resections were performed through posterolateral thoracotomy and two of them were performed by videoassisted thoracoscopic surgery. There were intraoperatively detected pleural adhesions around the lesions in all patients. In 2 patients a concomitant bronchogenic cyst was detected and resected in the same session. In one patient, there was solitary pulmonary nodule in the right upper lobe which sized in diameter as $1 \times 1.5 \mathrm{~cm}$ and have diffuse calcification. The postoperative pathological result was hamartoma.

One patient (3.2\%) with ELS had systemic blood supply originating from left subclavian artery (Figure 1), and the rest of the patients $(96.8 \%)$ had systemic blood supply originating from the aorta (Figure 2). One patient (3.2\%) had abdominal aortic branch as systemic blood supply. Three patients $(9.7 \%)$ had between 2 and 5 aortic branches and one patient had over 5 aortic branches as systemic blood supply.

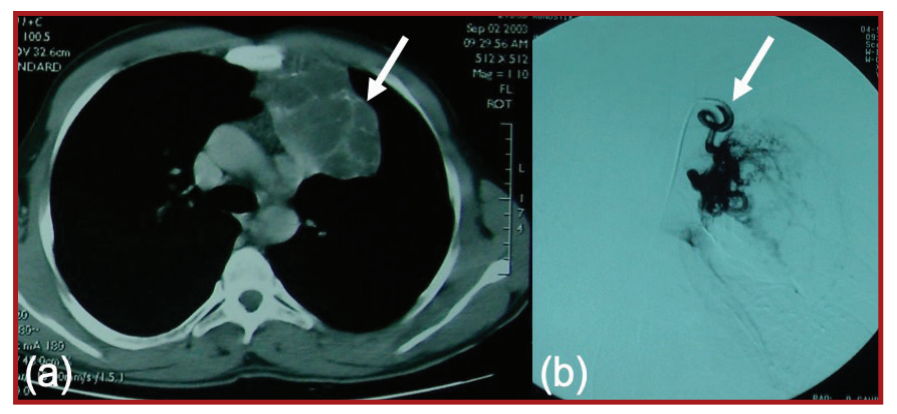

Figure 1. CT scan of the left ELS (a) and angiography of systemic blood supply originating from left subclavian artery (b).

Six patients had postoperative complications: prolonged air leak in 4 patients, pneumonia in 2 patients. Pneumonia treated with appropriate antibiotic treatment. During the follow up peri- 


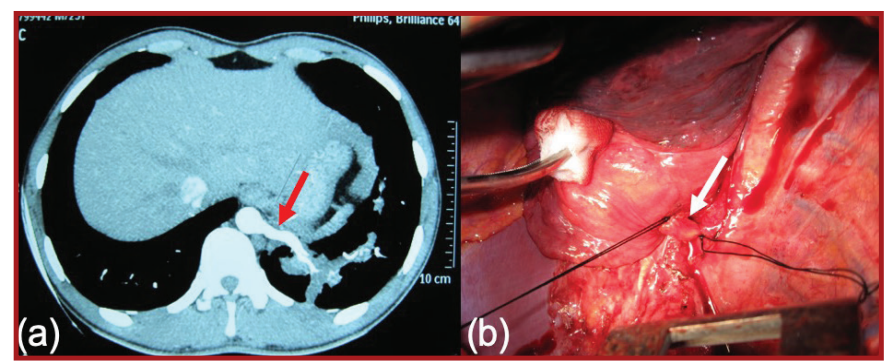

Figure 2. CTA scan of systemic blood supply originated from the aorta (a) and the intraoperative view of the systemic artery (b).

od ranging between 4 month and 13 years (mean 28.4 month), there was only one mortality because of myocardial infarction at the age of 54 after 4 years from the surgery.

\section{Discussion}

Pulmonary sequestration may remain silent in patients and can be diagnosed at an adult age (6). Adult patients usually manifest upper airway syndrome, hemoptysis, or repeated lung infections. Diagnosis is usually confirmed by imaging results based on the above symptoms. Surgical resection of the diseased lung, either lobar or sublobar resection, has been established as the definitive treatment for eliminating active symptoms and preventing the progression of airway complications (7).

The etiology of PS is still not clear but some theories have been suggested so far. The commonly accepted hypothesis is suggested by Corbett and Humphrey. Accordingly, a separate lung bud is formed during intrauterine growth and this bud is blooded from the systemic circulation (4). It is widely accepted that ELS has congenital origin, but the origin of ILS is controversial. The low incidence of congenital anomalies in ILS supports the idea of acquired etiology (8). According to this view, small systemic arteries in the pulmonary ligament invade the infected tissue as a result of continuous infection attacks (9).

ILS is seen about three-quarters of all cases $(10,11)$. In our series, number of the ELS and ILS cases were almost equal in numerical order. It has been reported that ELS is more common in men. ILS does not have such a frequency $(1,4)$. In our series, 15 of 16 ELS patients were male.

Extralobar sequestrations are separated from the ILS with their own visceral pleura. Extralobar sequestration is most commonly seen $(63 \%)$ between the diaphragm and the lower lobe. Intralobar sequestration is located in lower lobes with rate of $98 \%$ (4). Bilateral cases are found very rarely (12). Frequent occurrence of the PS in the lower lobes should be stimulant for clinicians. In our series of 31 patients, in 30 patients' sequestrations were located in the lower lobes and there were no bilateral cases. Atypical placements for ELS have been reported and congenital anomaly association is more frequent than ILS (11). The most common anomaly associations are; diaphragmatic hernia and defects, cardiopulmonary anomalies and foregut connection. In our series, we determined that ELS is located in the middle lobe in one patient. Two patients with bronchogenic cysts had ELS-type sequestration.

Wei et al. showed that $58.3 \%$ of cases were misdiagnosed in the preoperative diagnosis of sequestration in their series of 2625 patients (12). In differential diagnosis, malignancies such as abscess, pneumonia, hypoplasia, pericardial cyst, cystic adenoid malformation, congenital lobar emphysema, neurogenic tumor and lung cancer are prominent (13). This large differen- tial diagnosis list demonstrates the importance of preoperative rigorous evaluation.

Chest X-ray and computed tomography (CT) scan shows nonspecific images like mass, cystic, cavitary, or pneumonic lesions. For the diagnosis of PS, the presence of a feeding systemic artery must be proved (9). Imaging examination can clearly reveal aberrant feeding arteries, providing key information for preoperative diagnosis. Enhanced chest CT can clearly show aberrant feeding arteries in a noninvasive manner and has thus become the preferred examination for PS; it can also reveal the abnormal drainage veins. Magnetic resonance imaging can detect the relationship between the lesion and the aberrant feeding arteries of the systemic circulation without the use of contrast agents; however, it is less useful than enhanced CT in revealing the pathologic features of PS $(14,15)$. In our series, six of the patients were evaluated with CTA, one patient was evaluated with MRA in the preoperative period. It is stated in literature that anomalous systemic arterial supplies came from the descending thoracic aorta (72\%); the abdominal aorta, celiac axis, or splenic artery (21\%); the intercostal artery (3\%); and rarely the subclavian, left gastric, superior mesenteric, phrenic, and renal arteries (and their branches) or via the pericardium and coronary artery $(14,16)$. In our series, as it is very rare in the literature, one patient $(3.2 \%)$ with ELS had systemic blood supply originating from left subclavian artery (Figure 1), and the rest of the patients $(96.8 \%)$ had systemic blood supply originated from the descending aorta (Figure 2). One patient (3.2\%) had abdominal aortic branch as systemic blood supply. Three patients $(9.7 \%)$ had between 2 and 5 aortic branches and one patient had over 5 aortic branches as systemic blood supply.

All patients selected for this study were operated in our clinic and their diagnosis was confirmed perioperatively and confirmed by pathology. In two cases, we observed the bronchial relationship of PS to the main bronchi, intraoperatively, but we could not prove this radiologically because the imaging studies could not be reached due to technical problems. If this connection can be proved in the future studies, this connection may create a new subtype of congenital malformation.

Clinical findings are miscible with many other lung diseases and are not specific in the diagnosis of PS. Cough, sputum, fever, hemoptysis and chest pain are the most common symptoms (12). The most common symptom in our series was dyspnea $(n=10)$. Five patients had chest pain. Pulmonary sequestration may be asymptomatic or accidentally diagnosed. Seven of our cases were asymptomatic. Although patients can be diagnosed at any age, they are most frequently diagnosed in the first two decades (12). In our series, the mean age at diagnosis was 24.6 years.

Surgical resection of the diseased lung is recommended for symptomatic patients and should also be performed for some asymptomatic patients to control and prevent recurrent pulmonary infection by stopping the inflammatory process and to lower the incidence of airway complications (7). Medical treatment is effective only in the treatment of accompanying infections. In our series, sequestered mass was removed by cutting the systemic artery in all ELS cases. Thirteen of the ILS cases underwent lobectomy and two of them underwent segmentectomy. During the surgery, especially trans diaphragmatic abdominal aortic branches may cause dangerous situations. While dissection, rupture of an abdominal 
aortic branch may drop in to abdomen which can cause massive bleeding which needs urgent laparotomy.

The main purpose of surgery is to prevent complications. Because, fungal infection, tuberculosis, fatal hemoptysis, massive hemothorax and malignancy have been reported in untreated cases (17). In our series, 2 patients presented with hemoptysis. Embolization may be considered in certain cases that do not accept surgery or not suitable for medical condition (18).

\section{Conclusion}

Pulmonary sequestration is a rare disease in which some claims about etiology have been suggested. It has similar appearance with many diseases of lung with traditional imaging methods and cause difficulties in diagnosis. Proving the presence of a feeding systemic artery with angiographic imaging is essential for definitive diagnosis. Differential diagnosis is clinically important because untreated cases are open to complications. The recommended optimal treatment for pulmonary sequestration is resection of the sequestered tissue by segment or lobar resection.

\section{Acknowledgments}

Authorship contributions: Concept: E.S.; Design: E.S., M.Ş.I., H.I.; Supervision: S.G., H.Ç.; Resources: E.S., O.K., H.Ç., S.G.; Materials: H.I., O.K.; Data collection and/or processing: E.S., M.Ş.I.., H.I., O.K.; Analysis and/or interpretation: E.S., M.Ş.I.., H.I., H.Ç.; Writing: M.Ş.İ., E.S., H.Ç.; Critical review: S.G.

\section{Conflict of Interest}

The authors declared they do not have anything to disclose regarding conflict of interest with respect to this manuscript.

\section{References}

1. Frazier AA, Rosado de Chistenson ML, Stocker J. Intralober sequestration: radiolojic- patholojic correlation. Radiographics. 1997;17(3):725-745.

2. Pratter MR, Kaemmerlen JT, Ericson AD. Bloody pleural effusion associated with an intralobar pulmonary sequestration. Chest 1979;75(3):394-395.

3. Jeanfaivre T, Afi M, L'Hoste P. Simultaneous discovery of bilateral intralobar and exralobar pulmonary sequestrations. Ann Thorac Surg 1997;63(4):1171-1173.

4. Corbett HJ, Humphrey GM. Pulmonary sequestration. Pediatr Respir Rev 2004;5(1):59-68

5. Yucel O, Gurkok S, Gozubuyuk A, et al, Diagnosis and surgical treatment of pulmonary sequestration. Thorac Cardiovasc Surg. 2008;56(3):154-157.

6. Van Raemdonck D, De Boeck K, Devlieger $\mathrm{H}$, et al. Pulmonary sequestration: a comparison between pediatric and adult patients. Eur J Cardiothorac Surg 2001;19(4):388-395.

7. Lin TH, Huang WL, Chang CC, et al. Uniportal video-assisted thoracoscopic surgery lobectomy and segmentectomy for pulmonary sequestration. J Thorac Dis 2018;10(6):3722-3728

8. Stocker JT, Malczak HT. A study of pulmonary ligament arteries. Relationship to intralobar pulmonary sequestra- tion. Chest. 1984;86(4):611-615.

9. Bolca N, Topal U, Bayram S. Bronchopulmonary sequestration: radiologic findings. Eur J Radiol 2004; 52(2):185-191

10. Lagauise P, Bonnard A, Berrebi D, et al. Video-assisted thoracoscopic surgery for pulmonary sequesrrstion in children. Ann Thorac Surg. 2005;80(4):1266-1269.

11. SavicB, BirtelFJ, TholenW. Lungsequestration:report of seven cases and review of 540 published cases. Tho$\operatorname{rax} 1979 ; 34(1): 96-101$.

12. Wei Y, Li F. Pulmonary sequestration: a retrospective analysis of 2625 cases in China. Eur J Cardiothorac Surg 2011;40(1):39-42.

13. Cooke CR. Bronchopulmonary sequestration. Respir Care 2006;51(6):661-664.

14. Zhang N, Zeng Q, Chen C, Yu J, Zhang X. Distribution, diagnosis, and treatment of pulmonary sequestration: Report of 208 cases, J Pediatr Surg (2018), https://doi. org/10.1016/j. jpedsurg.2018.08.054.

15. Walker CM, Wu CC, Gilman MD, et al. The imaging spectrum of bronchopulmonary sequestration. Curr Probl Diagn Radiol 2014;43(3):100-114.

16. Laborda A, Ruiz JR, De Gregorio MÁ. Congenital pulmonarysequestration supplied by the right coronary artery. Arch Bronconeumol 2016;52(7):389.

17. Van Raemdonck D, De Boeck K, Devlieger $H$, et al. Pulmonary Sequestration: a comparison between pe- diatric and adult patinets. Eur J Cardiothorac Surg 2001;19(4):388-395.

18. Yucel O, Caylak H, Gozubuyuk A, et al. Use of embolization and surgical resection in a case of pulmonary sequestration manifesting with hemopthysis. Turk Toraks Dergisi 2011;12(4):172-175. 